\title{
Photophysics of polymeric carbon nitride: An optical quasimonomer
}

\author{
C. Merschjann, ${ }^{1,2, *}$ T. Tyborski, ${ }^{1,3}$ S. Orthmann, ${ }^{1,4}$ F. Yang, ${ }^{1}$ K. Schwarzburg, ${ }^{1}$ M. Lublow, ${ }^{1,5}$ \\ M.-Ch. Lux-Steiner, ${ }^{1}$ and Th. Schedel-Niedrig ${ }^{1}$ \\ ${ }^{1}$ Helmholtz-Zentrum Berlin für Materialien und Energie GmbH, D-14109 Berlin, Germany \\ ${ }^{2}$ Universität Rostock, D-18055 Rostock, Germany \\ ${ }^{3}$ Max-Born-Institut für Nichtlineare Optik und Kurzzeitspektroskopie, D-12489 Berlin, Germany \\ ${ }^{4}$ Technische Universität Berlin, D-10623 Berlin, Germany \\ ${ }^{5}$ Leibnitz-Institut für Katalyse e.V., D-18059 Rostock, Germany
}

(Received 5 October 2012; revised manuscript received 6 November 2012; published 15 May 2013)

\begin{abstract}
A comprehensive investigation of the luminescent properties of carbon nitride polymers, based on tri-s-triazine units, has been conducted. Steady-state temperature- and excitation-power-dependent as well as time-resolved measurements with near-UV excitation $(\lambda=325 \mathrm{~nm}$ and $405 \mathrm{~nm}$ ) yield strong photoluminescence, covering the visible spectrum. The spectral, thermal, and temporal features of the photoluminescence can be satisfactorily described by the excitation and radiative recombination of molecular excitons, localized at single tri-s-triazine units. The discussed model is in accordance with the recently reported absorption features of carbon nitride polymers. Thus, from the point of view of optical spectroscopy, the material effectively behaves as a monomer.
\end{abstract}

DOI: 10.1103/PhysRevB.87.205204

\section{INTRODUCTION}

Polymeric carbon nitrides (PCN) have been successfully applied for photocatalytic hydrogen and oxygen production from water under visible light, both as pristine powder ${ }^{1}$ and as nanocrystalline films in heterojunction photocathodes. ${ }^{2}$ However, the origin of PCN's photocatalytic activity could not be revealed to date. This is in part due to the fact that many basic physical characteristics of PCN are either unknown or bear large uncertainties. These include especially the crystal and electronic structure of the materials. The ambiguity concerning the crystal structure is partly caused by the variety of different synthesis routes, leading to more or less well-defined materials. ${ }^{3-8}$ The most prominent members of the class are Melem $\left(\mathrm{C}_{6} \mathrm{~N}_{10} \mathrm{H}_{6}\right),{ }^{9}$ Melon $\left(\mathrm{C}_{6} \mathrm{~N}_{9} \mathrm{H}_{3}\right),{ }^{5}$ and graphitic carbon nitride $\left(\mathrm{g}-\mathrm{C}_{3} \mathrm{~N}_{4}\right){ }^{3}$ While all of these composites share the tri-s-triazine unit as their basic building block, in practical cases it is often hard to determine the actual composition and structure of the compound. Thus it is most likely that the majority of the common synthesis processes result in a structure best described as Melon in different stages of condensation. ${ }^{5}$ We will take an idealized structure of Melon-based PCN, as suggested by Lotsch et al. and shown in Fig. 1(a), ${ }^{5,10}$ as the basis of discussion throughout this work.

Concerning the electronic structure, it has often been assumed that PCN behaves like a semiconductor, since densityfunctional calculations gave hints in this direction. ${ }^{1,11}$ From the experimental point of view, a pronounced optical band gap in the blue spectral range around $2.7 \mathrm{eV}$ was deduced from diffuse reflectance spectra. ${ }^{1,4} 8$ It was furthermore observed that these optical transitions are tunable by changing the temperature of the PCN synthesis process, ${ }^{1,4,8,12}$ giving evidence for a dominant transition within the tri-s-triazine unit

Published by the American Physical Society under the terms of the Creative Commons Attribution 3.0 License. Further distribution of this work must maintain attribution to the author(s) and the published article's title, journal citation, and DOI.
PACS number(s): 78.55.Kz, 77.84.Jd, 42.70.Jk, 82.65.+r

of the polymer. ${ }^{12}$ At the same time, photoluminescence (PL) experiments revealed quite strong emissions, covering almost the whole visible range, and corresponding to the absorbance spectra. ${ }^{4,13,14}$

Despite these evidences, a thorough insight into the optical absorption and emission process-and, thus, the photophysics - in PCN is still missing. This is, however, one of the crucial points concerning the nature of PCN's photocatalytic activity.

Unfortunately, there exists no method to date to produce PCN samples of at least near-optical quality (e.g., thin films, transparent bulk crystals, or solutions). Thus a quantitative determination of linear optical measures like absorption coefficient $\alpha$ and refractive index $n$ as functions of the light wavelength is virtually impossible. Moreover, the available highly scattering samples impede standard techniques used to distinguish between semiconductor and molecular optical materials, such as two-photon-absorption and electroabsorption spectroscopy. ${ }^{15,16}$

On the other hand, PL spectroscopy provides a relatively simple and robust method to determine the optical properties of semiconductors. ${ }^{17}$ Although in general no quantitative figures of the spectroscopic measures can be extracted, their time, temperature, and excitation-power dependence is sufficient to deduce the nature of optical excitation and emission processes, including energy levels of excitons and point defects. Given the experimental obstacles mentioned above, it is reasonable to revert to PL spectroscopy in order to shed light on the photophysical behavior of PCN, especially with respect to the question whether PCN is better described as a semiconductor or a molecular system.

In this work we present an investigation of the PL of PCN in various states of condensation. The spectral, thermal, and temporal features of the PL can be satisfactorily described by the excitation and radiative recombination of molecular excitons, localized at single tri-s-triazine units. The discussed model is in accordance with the recently reported absorption features of PCN. ${ }^{12}$ It is therefore suggested that, at least from the point of view of optical spectroscopy, PCN effectively 
(a)

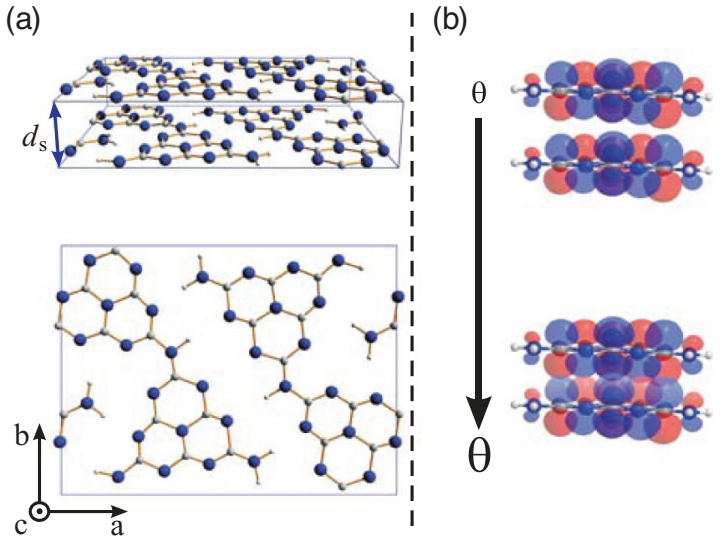

FIG. 1. (Color online) (a) Idealized structure of PCN, based on Melon strands in an orthorhombic unit cell (after Lotsch et al.). ${ }^{5}$ The interplanar stacking distance $d_{\mathrm{s}}$ is depicted. (b) Sketch of the effect of higher process temperatures on the overlap of $\pi$ orbitals of mutual tri-s-triazine units along the stacking direction. Note that the effect has been exaggerated for better visibility.

behaves as a monomer, in contrast to the classical semiconductor conjecture.

\section{EXPERIMENTAL DETAILS}

Dicyandiamide (Aldrich, 99\% purity, about $2.5 \mathrm{~g}$ ) was heated at a rate of $25 \mathrm{~K} / \mathrm{min}$ in a ceramic crucible within a muffle furnace under continuous $\mathrm{N}_{2}$ gas flow to a temperature in the range $400^{\circ} \mathrm{C} \leqslant \theta \leqslant 610^{\circ} \mathrm{C}$. ${ }^{12}$ In order to achieve homogeneous processing, the materials were pestled shortly after the first thermal treatment. The resulting products were ground and additionally annealed at the same temperature for another $3 \mathrm{~h}$ under $\mathrm{N}_{2}$ gas flow, whereafter they were allowed to cool down passively within the furnace. The specific process temperatures have been chosen to represent the regime of polycondensation of tri- $s$-triazine units $\left(\approx 500{ }^{\circ} \mathrm{C}-600^{\circ} \mathrm{C}\right) .{ }^{4}$ A sample processed at $400{ }^{\circ} \mathrm{C}$ served for reference purposes, since a high content of the tri-s-triazine monomer can be expected. ${ }^{4,9}$ The crystal structure was investigated using $\mathrm{X}$-ray diffraction; the samples under study showed the characteristic features of PCN (see Tyborski et al. for details). ${ }^{10,12}$

Steady-state PL spectra were recorded using the custombuilt setup described elsewhere. ${ }^{18}$ The powder samples were fixed on a copper plate using rubber cement and mounted inside a He flow cryostat, allowing for temperatures in the range of $3.5 \mathrm{~K} \leqslant T \leqslant 320 \mathrm{~K}$, equipped with spectrosil B windows. PL was excited with the light of a He-Cd laser $(\lambda=325 \mathrm{~nm})$, and emission was collected with parabolic mirrors and directed to a Czerny-Turner spectrograph with attached Si-CCD camera. The recorded spectra were calibrated using a $\mathrm{Hg}$-line lamp and a calibrated tungsten halogen light source, respectively, as described by Merschjann et al. ${ }^{18}$

Time-resolved PL data were acquired via the time-resolved single photon counting technique (TRSPC) using a pulsed laser diode emitting at $\lambda=405 \mathrm{~nm}$ with a repetition rate of $1 \mathrm{MHz}$ as light source and a Si Geiger mode APD as photodetector. The temporal response function of the instrument was $100 \mathrm{ps}$ full width at half maximum (FWHM). The PL emission was separated by a dielectric $500 \mathrm{~nm}$ cutoff longpass filter. Two

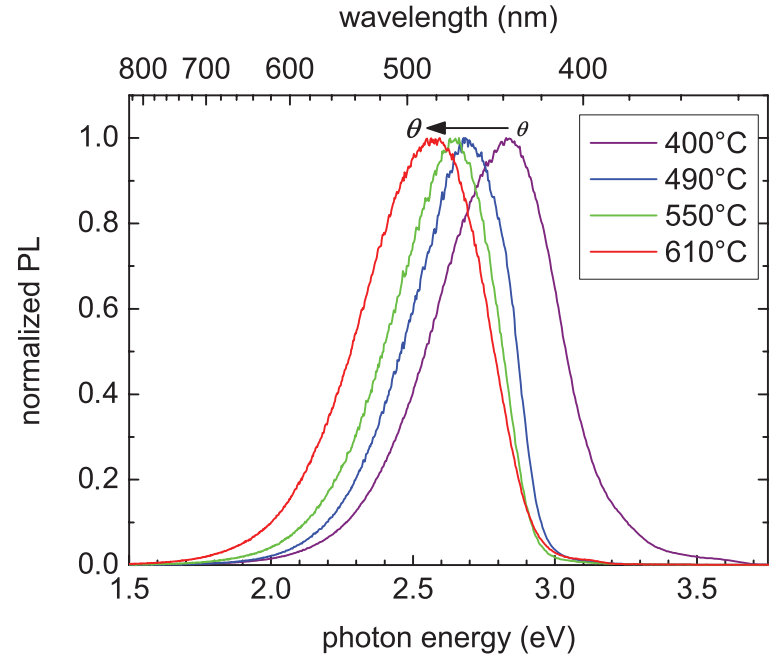

FIG. 2. (Color online) Normalized PL spectra of selected PCN powders taken at a temperature of $4 \mathrm{~K}$ and an excitation power of $8 \mathrm{~mW}$. The redshift with higher process temperature $\theta$ is clearly visible.

different average excitation powers of $15 \mu \mathrm{W}$ and $50 \mathrm{nW}$, respectively, were used in the experiments.

\section{RESULTS}

\section{A. Steady-state PL}

Figure 2 shows normalized PL spectra of four selected PCN powders, recorded at a temperature of $4 \mathrm{~K}$ with an excitation power of $8 \mathrm{~mW}$. The typical features of the PCN luminescence are visible, comprising wide peaks in the UV-VIS spectral range. Furthermore, the already reported redshift with higher polycondensation temperature is evident. ${ }^{4,12}$ One typical characteristic of PCN photoluminescence is its asymmetry on the energy scale. This is shown in Fig. 3 exemplarily for a sample processed at $550^{\circ} \mathrm{C}$. Shown in black is the absorption coefficient $\alpha$, which has been estimated from the attenuance $A$ of a KBr/PCN mixed tablet. ${ }^{12}$ Here, a simple model is used, assuming the relation

$$
A=1-\exp [-\alpha d],
$$

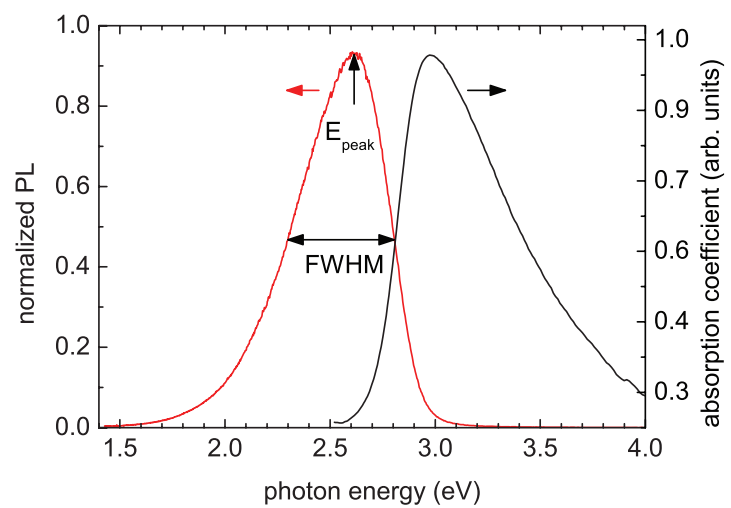

FIG. 3. (Color online) PL spectrum of a sample processed at $550{ }^{\circ} \mathrm{C}$, taken at room temperature with an excitation power of $8 \mathrm{~mW}$. Additionally, the absorption coefficient of a $\mathrm{KBr} / \mathrm{PCN}$ mixed tablet is shown (see Tyborski et al. ${ }^{12}$ ). 
whereby the optical path length $d$ depends on the light wavelength according to a Rayleigh-scattering model: $d \propto \lambda^{-4}$. Hence scattering from subwavelength sized PCN powder particles increases the apparent attenuance drastically in the UV range. One can estimate a formula for the absorption coefficient:

$$
\alpha \propto-\left(\frac{\lambda_{0}}{\lambda}\right)^{-4} \ln [1-A] .
$$

It can be seen that the steep drop of the PL at the high-energy side corresponds to the increase in the absorption spectrum. This indicates that the asymmetry is caused mainly by selfabsorption within the PCN material. In addition, there is a hint that indeed absorption and emission of the samples are mirrored about the energy scale, a feature common for molecular systems. ${ }^{19}$

Nevertheless, a proper quantitative analysis of the PL peaks is impeded, since the absolute value of the absorption coefficient is unknown and cannot be directly deduced from the above-given estimation. ${ }^{12}$ We will therefore apply the following approach in order to further analyze our spectra. The FWHM and the energetic peak position are defined as depicted in Fig. 3. Care needs to be taken upon transformation of the spectra from the wavelength to the photon energy scale, especially when fitting of the signals with, e.g., Gaussian functions is attempted, or even deconvolution of several emission bands needs to be done. In the case at hand, we are only interested in the integrated PL intensity, which can safely be calculated as the cumulative sum of the respective PL spectrum over either wavelength or energy scale. Using these characteristic values, we will now analyze the PL spectra with respect to excitation light power, sample temperature, and process temperature.

The excitation-power dependence of the PL intensity and peak position is shown in Fig. 4 for a sample processed at $550^{\circ} \mathrm{C}$. Spectra were taken at room temperature and at $4 \mathrm{~K}$, respectively. The normalized PL intensity at $4 \mathrm{~K}$ exhibits a power-law behavior with increasing excitation power, revealing an exponent $k=0.98 \pm 0.01$ over the whole range. The dashed line represents the same slope value, $k$, applied to the room-temperature data. One may readily observe the deviation for higher excitation powers $(P \geqslant 1 \mathrm{~mW})$. A similar behavior is found for the PL peak positions in Fig. 4(b). At low temperature the peak energy increases exponentially, with a rate of $14 \pm 2 \mathrm{meV}$ per decade. At room temperature, this general trend is also observed, but a distinctive exponential behavior cannot be found.

All steady-state PL spectra were also measured at different temperatures throughout the range 4-293 K. The results are exemplarily shown in Fig. 5 for the sample processed at $550^{\circ} \mathrm{C}$. No decisive trends can be identified. This is true especially for the integrated PL intensity, which scatters by a factor of about 3 within the temperature range, but does not show the typical features of thermal quenching, as commonly found for semiconductors.

We therefore turn our attention to an overview of the characteristic PL features found for the samples processed at different synthesis temperatures. Figure 6 shows the PL peak position and the FWHM as a function of process temperature. In addition, Fig. 6(a) includes the energies for the direct

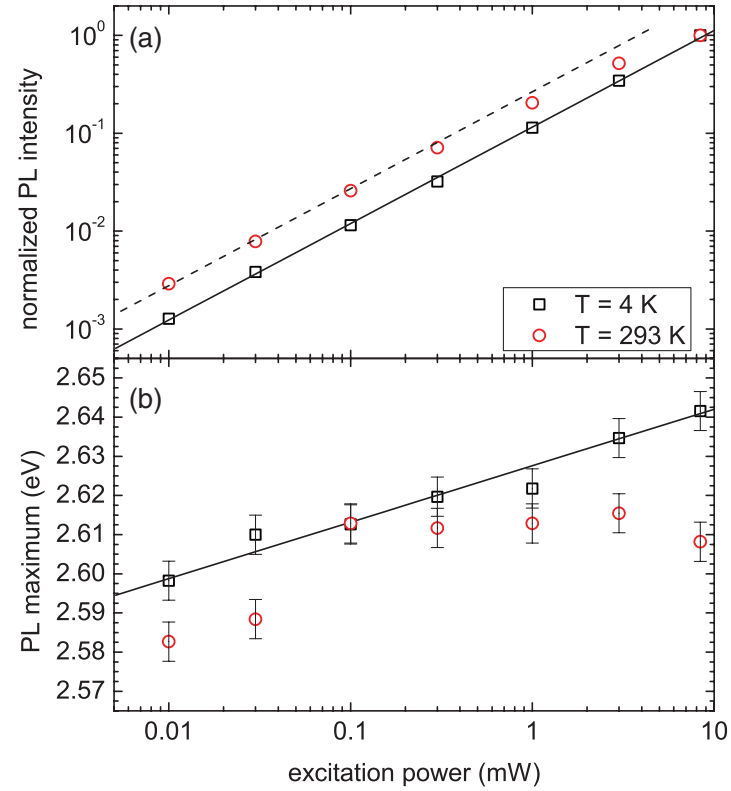

FIG. 4. (Color online) Excitation-power dependence of the integrated PL intensity (a) and of the PL peak energy (b) for a sample processed at $550{ }^{\circ} \mathrm{C}$, taken at room temperature and at $4 \mathrm{~K}$. The slope of the lines in (a) is 0.98 .

optical transition, as determined recently. ${ }^{12}$ Both absorptive transition and PL maxima decrease in energy with higher

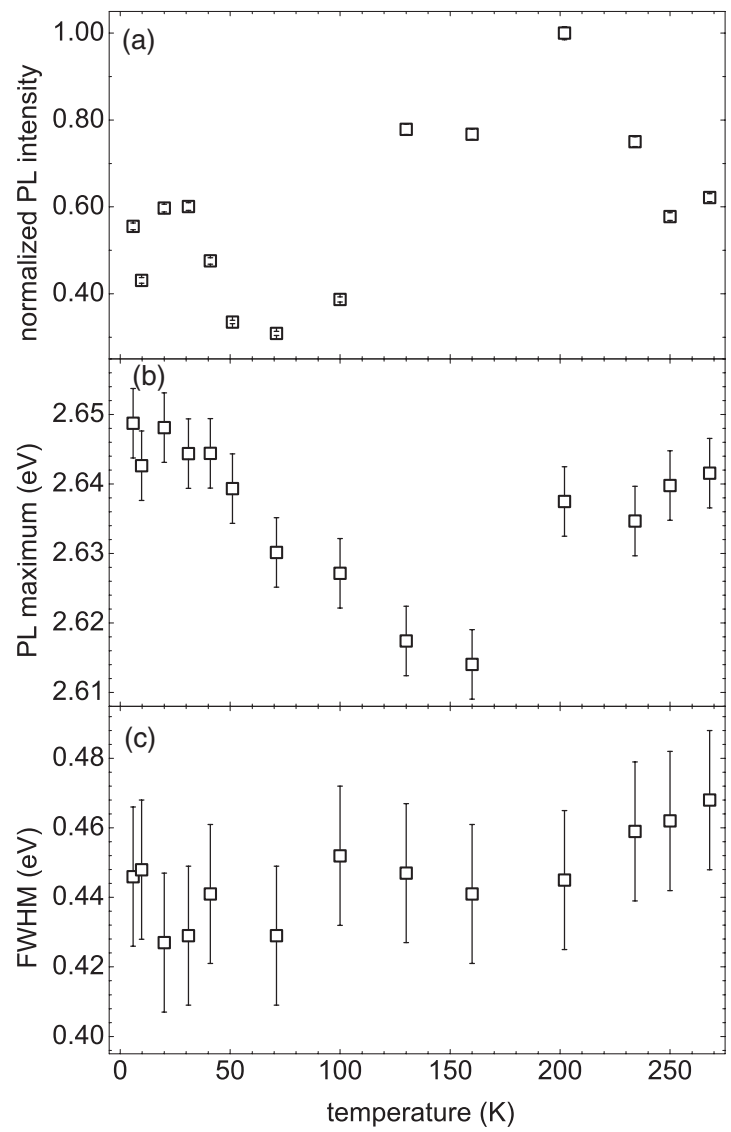

FIG. 5. Temperature dependence of the integrated PL intensity (a), the PL peak energy (b), and of the FWHM (c) for a sample processed at $550{ }^{\circ} \mathrm{C}$, taken at an excitation power of $8 \mathrm{~mW}$. 


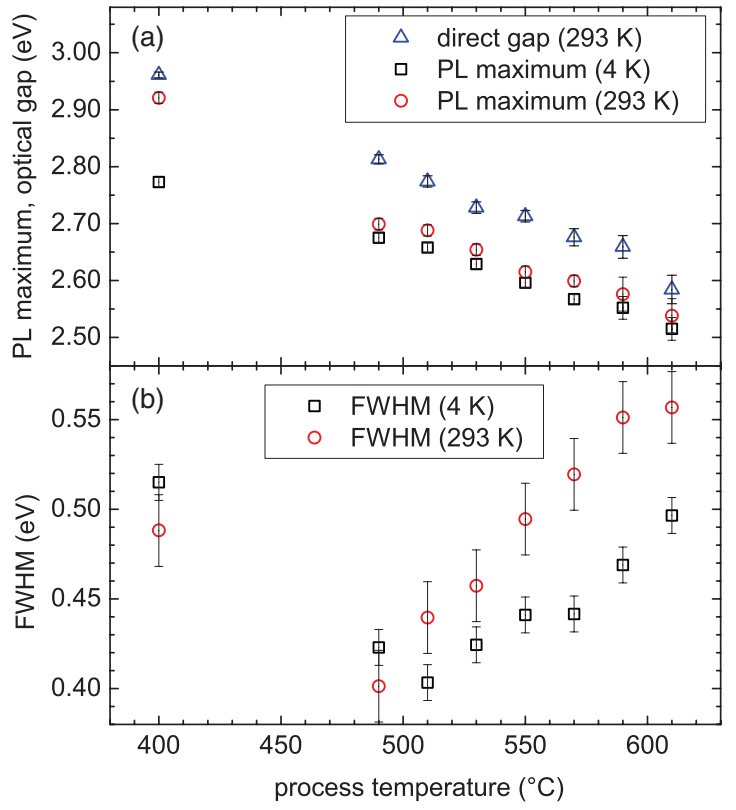

FIG. 6. (Color online) Process-temperature dependence of the PL peak energy (a) and the FWHM (b), taken at room temperature and at $4 \mathrm{~K}$. The excitation power is $0.01 \mathrm{~mW}$ in both cases. Additionally, the values for the direct optical transition at room temperature are depicted as triangles. ${ }^{12}$

process temperatures. At room temperature, the PL maxima lie about $70 \mathrm{meV}$ lower than the direct optical transition. On the other hand, the FWHM tendentially increases with higher process temperatures, as shown in Fig. 6(b). A substantial deviation from this general behavior is found for the sample processed at $400{ }^{\circ} \mathrm{C}$.

\section{B. Time-resolved PL}

All time-resolved PL measurements have been conducted at two excitation light powers (50 $\mathrm{nW}$ and $15 \mu \mathrm{W})$. In addition to the spectrally integrated signal used here, the PL was also recorded using a grating monochromator. No difference in the decay shapes could be found in dependence on either laser power or PL spectral range. The data shown here are for a laser power of $15 \mu \mathrm{W}$.

Figure 7 shows normalized time-resolved PL signals of four selected samples, recorded at room temperature. In all cases, the decay curves are strongly nonexponential, indicating a broad distribution of lifetimes of the excited electronic states. This behavior may be attributed to diffusive transport phenomena of either energy or particles (electrons, holes, or excitons). Since well-founded theoretical models for transport in PCN are not available to date, we will stick to phenomenological descriptions for the time being. One commonly used description is given by the stretched exponential decay according to Kohlrausch, Williams, and Watts: ${ }^{20}$

$$
f_{\mathrm{KWW}}(t)=A_{0} \exp \left[-\left(t / \tau_{\mathrm{KWW}}\right)^{\beta}\right],
$$

with the initial amplitude $A_{0}$, mean lifetime $\tau_{\mathrm{KWw}}$, and stretching exponent $0<\beta \leqslant 1$. Besides the mean lifetime, its average (or expectation) value is often used, as

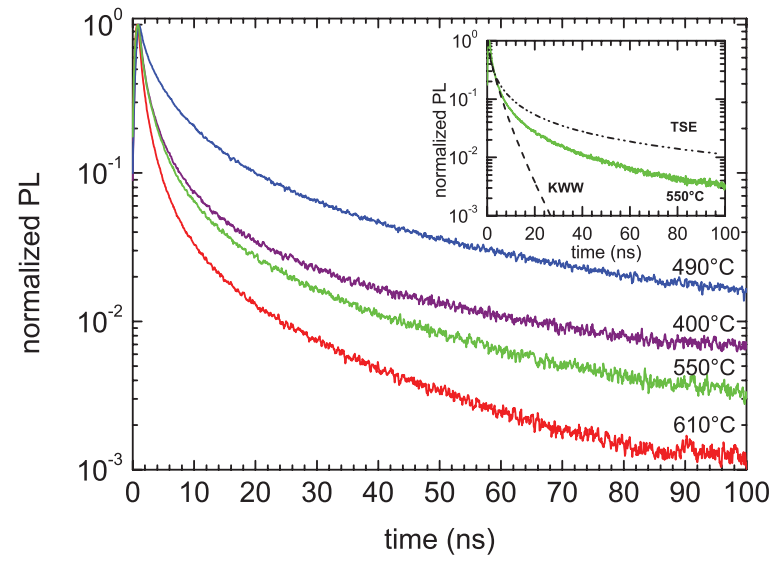

FIG. 7. (Color online) Normalized PL decay curves of selected PCN powders taken at room temperature. The inset shows fits by Eqs. (3) and (5), depicted by the dashed and dashed-dotted line, respectively. The relevant fit parameters are shown in Fig. 8.

defined by ${ }^{21}$

$$
\left\langle\tau_{\mathrm{KWW}}\right\rangle=\frac{\tau_{\mathrm{KWW}}}{\beta} \Gamma\left[\frac{1}{\beta}\right],
$$

where $\Gamma[x]$ is the gamma function.

Another approach may be to assume a charge separation during the excitation process. The recombination can in this case be described in terms of a bimolecular reaction or, seen from the point of view of solid-state physics, as a trap-saturation effect (TSE): ${ }^{22}$

$$
f_{\mathrm{TSE}}(t)=\frac{A_{0}}{1+t / \tau_{\mathrm{TSE}}} .
$$

Again, $A_{0}$ denotes the initial amplitude, while $\tau_{\mathrm{TSE}}$ is the halflife of the TSE decay.

Both models are applied to fit the data, the results being shown in the inset of Fig. 7 exemplarily for the sample processed at $550{ }^{\circ} \mathrm{C}$. The fits are not very good for $t>10 \mathrm{~ns}$, over- or underestimating the data, respectively. This indicates that the "real" process is indeed more complex than what is described by Eqs. (3) and (5). However, a preliminary analysis can be done. This is shown in Fig. 8, where the mean and average lifetimes are plotted versus the process temperature. The stretching exponent $\beta$ is given in the inset. While the data for $400{ }^{\circ} \mathrm{C}$ apparently deviate from those for the higher process temperatures (cf. Fig. 6), the latter show a more systematic behavior. This is true for both $\beta$ and $\tau$, the former increasing roughly linearly with a slope of $2.1(3) \times 10^{-3} \mathrm{~K}^{-1}$. All fitted lifetimes decrease nonlinearly with the process temperature $\theta$. Without a specific model, one may fit the data in the $490{ }^{\circ} \mathrm{C}-610^{\circ} \mathrm{C}$ range with an exponential decay:

$$
\tau(\theta)=\tau_{\text {res }}+\tau_{\max } \exp \left[-\frac{\theta-490^{\circ} \mathrm{C}}{\theta_{0}}\right],
$$

with the decay parameter $\theta_{0}$, and the maximum and residual lifetimes $\tau_{\max }$ and $\tau_{\text {res }}$, respectively. The results are shown in Table I. It is evident that, although the lifetimes for the different models differ by roughly a factor of 2 , the decay parameter is the same within numerical accuracy. 


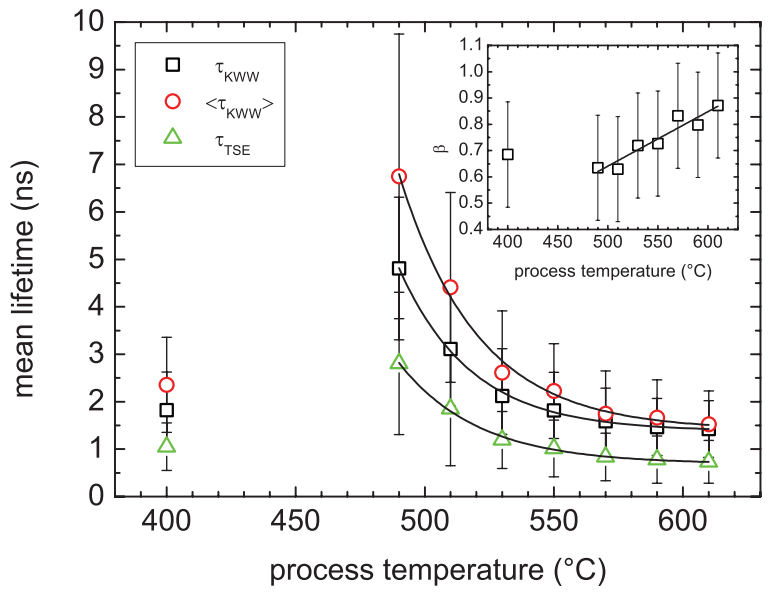

FIG. 8. (Color online) Parameters obtained from fits of Eqs. (3) and (5) to the data shown in Fig. 7. The lines are fits of Eq. (6) to the data. For details, see the text.

\section{DISCUSSION}

As mentioned above, polymeric carbon nitride has often been described as a semiconductor. However, as we will see in the following, this conjecture is not supported by the experimental data presented in this work. We will show instead that the results of our optical investigations can be consistently described within the context of a molecular system. It is in fact found that the PL results obtained here closely resemble those commonly observed in conjugated polymers. ${ }^{23}$ Recently, we have shown that the optical absorption of PCN is most likely dominated by a direct transition within the tri- $s$-triazine unit (the monomer of PCN). ${ }^{12}$ According to the results of our PL investigation, we can now identify this transition with the optical generation of molecular excitons, most probably of singlet nature. Following this hypothesis, we are able to explain our results in more detail.

\section{A. Spectral shape of absorption and emission}

As can be seen in Figs. 2 and 3, the PL emission is spectrally asymmetric, suggesting a quite strong self-absorption. At the same time, absorption and emission profiles are nearly mirror images, a feature typical for molecular excitons. ${ }^{24}$ The observed spectral bandwidth (FWHM) of about $500 \mathrm{meV}$ is quite large, compared to that of common conjugated polymers $(\approx 100 \mathrm{meV}),{ }^{23}$ and especially to those of wideband-gap semiconductors (e.g., GaN: FWHM $<2 \mathrm{meV}){ }^{25}$ However, typical excitonic absorption and emission in conjugated polymers shows vibronic replicas according to a Franck-Condon progression. ${ }^{23,24}$ In combination with a high phonon energy (>100 meV), a fairly broad, though structured,

TABLE I. Parameters obtained from fits of Eq. (6) to the lifetime data shown in Fig. 8.

\begin{tabular}{llll}
\hline \hline Model & $\tau_{\max }(\mathrm{ns})$ & $\tau_{\text {res }}(\mathrm{ns})$ & $\theta_{0}\left({ }^{\circ} \mathrm{C}\right)$ \\
\hline$\tau_{\mathrm{KWW}}$ & $3.44(6)$ & $1.38(4)$ & $28(1)$ \\
$\left\langle\tau_{\mathrm{KWW}}\right\rangle$ & $5.4(2)$ & $1.4(1)$ & $31(3)$ \\
$\tau_{\mathrm{TSE}}$ & $2.13(6)$ & $0.68(4)$ & $31(2)$ \\
\hline \hline
\end{tabular}

absorption/emission profile is expected..$^{23} \mathrm{~A}$ possible reason why we observe an apparently featureless PL may be the highly undefined crystal structure of PCN, resulting in a large degree of disorder. As a consequence, a distribution of energy levels occurs, causing a broadening of the PL bandwidth. ${ }^{23,26}$ Broadening is further enhanced since the excitonic bandwidth is proportional to the intermolecular coupling, which in turn is roughly inversely proportional to the distance between mutual monomer units. ${ }^{23,26-28}$

\section{B. Excitation-power and temperature dependence}

The PL emission intensity exhibits a linear dependence on the excitation light power over several decades. Following the molecular-exciton hypothesis, this observation can be readily explained. ${ }^{29}$ One has to keep in mind that PCN is not a conjugated polymer, since the monomers are connected via nonaromatic nitrogen atoms along the Melon strands (see Fig. 1). ${ }^{3,4,11}$ As a consequence, photogenerated carriers are highly confined to their original tri-s-triazine unit, where at most one exciton may reside at a given time. ${ }^{11}$ While these excitons may in principle be split thermally to yield free electron-hole pairs, the required activation energy (i.e., the exciton binding energy) is usually quite high, in the range of several hundred meV. ${ }^{24}$ Although a direct determination of the binding energy is often impossible, we may deduce from the absence of thermal quenching in our samples (cf. Fig. 5) an activation energy in excess of about $200 \mathrm{meV}$, well above the room temperature equivalent of $25 \mathrm{meV}$. Slight deviations from the linear $I-P$ dependence at ambient temperature may be attributed to thermally activated nonradiative channels, such as exciton migration and subsequent nonradiative recombination at so far unidentified defects in the PCN structure (see Sec. IV C).

Clark et al. have reported on a blueshift and broadening of the emission spectra of regioregular poly(3-hexylthiophene). ${ }^{23}$ In our case, we do not observe a decisive blueshift; at the same time only a slight increase in emission bandwidth upon heating the samples to room temperature is evident. Whether this finding has to be attributed to competing light-induced electronic processes or the structural disorder within the investigated PCN samples needs to be further clarified.

While the PL emission seems as a whole unaffected by increasing temperatures, a comparably strong blueshift with higher excitation power is observed, which substantially exceeds values commonly obtained in inorganic semiconductors $(\approx 1-3 \mathrm{meV} / \mathrm{dec}) .{ }^{17,18}$ There is to date no consistent explanation for this finding, although one may speculate that, e.g., the $0-0$ transitions could be enhanced at higher excitation powers due to processes yet to be identified.

\section{Time dependence and transport phenomena}

As we could see, the temporal development of the PL emission is highly nonexponential, pointing to a fairly complex relaxation behavior, which cannot be satisfactorily described by the two commonly applied approaches used in our analysis. This is most probably caused by the fact that the PL is not simply proportional to the population density of excited states (in this case molecular excitons), but is affected by 
the interactions between radiative and nonradiative relaxation processes, whose characteristics are so far unknown for PCN. ${ }^{30}$ However, both analytic approximations yield mean lifetimes for the PL emission in the range of a few nanoseconds, which are quite common for singlet excitons in organic molecules and polymers. ${ }^{23,29}$ Concerning the decay shapes the reader should note that similar nonexponential decays have been found in other organic polymers as well. ${ }^{29}$ While a bimolecular recombination of electrons and holes according to Eq. (5) is unlikely for reasons mentioned earlier, there may still be a spatial migration of charge-neutral excitations, either as energy (e.g., Förster resonant energy transfer) or in the form of the molecular excitons themselves. ${ }^{29}$ The former would yield an $r^{-6}$ dependence on the spatial distance $r$ between excitation and emission loci. ${ }^{31,32}$ The latter is generally governed by hopping transport, ${ }^{29}$ the more so as the energy barrier between the tri-s-triazine units along the strands is high $(>1 \mathrm{eV}) .{ }^{11}$ However, a general analytical description of hopping transport is in most cases impossible, and one has to turn to numerical approaches, e.g., random-walk simulations. ${ }^{22,29}$ These analyses depend crucially on the assumptions made and a thorough investigation is beyond the scope of this initial work. One commonly used model is that of an exponentially decreasing transfer rate with increasing distance between hopping sites, in our case the monomer units. ${ }^{29}$ As we will see in the following section, this assumption may be well justified in the case under study.

\section{Process-temperature dependence and a qualitative model}

In contrast to the ambient (i.e., PL-experiment) temperature, we find that the process temperature has a decisive impact on the absorption and emission behavior of the PCN samples studied. It has been shown earlier that there is a dependence of the PCN crystal structure on the synthesis temperature, which is especially obvious in the more or less linear decrease of interplanar stacking distances $d_{\mathrm{s}}$ between the PCN sheets. ${ }^{4,12}$ We may therefore translate our process temperatures to stacking distances in the polycondensation regime between $490^{\circ} \mathrm{C}$ and $610^{\circ} \mathrm{C}$, as shown in the inset of Fig. 9. Due to the poor crystallinity of the materials, the error margins are comparably large, so the assignments made in the following should be considered as qualitative assessment only. A more detailed investigation of the crystal structure is currently in preparation. ${ }^{10}$

However, the general trends are clearly observable, and can be assigned to the excitation and relaxation of molecular excitons on tri-s-triazine units within PCN. The cofacial distance is expected to play a decisive role, since the interactions between $\pi$-conjugated molecules depend crucially on the mutual overlap of their orbitals. ${ }^{33}$ At the same time, the interaction along the polymer strand is probably weak due to the nonaromatic connections between the monomer units. ${ }^{11}$ Therefore, we concentrate here on the interplanar stacking distance $d_{\mathrm{s}}$ as the relevant model parameter. Figure 1(a) shows an idealized PCN structure based on Melon strands, as discussed by Lotsch et al. ${ }^{5}$ For reasons of simplicity, an orthorhombic unit cell with $A-A$ stacking (distance $d_{\mathrm{s}}$ ) is assumed, where the one-dimensional Melon strands are stabilized by hydrogen bonds in the $a$ - $b$ plane, while the sheets

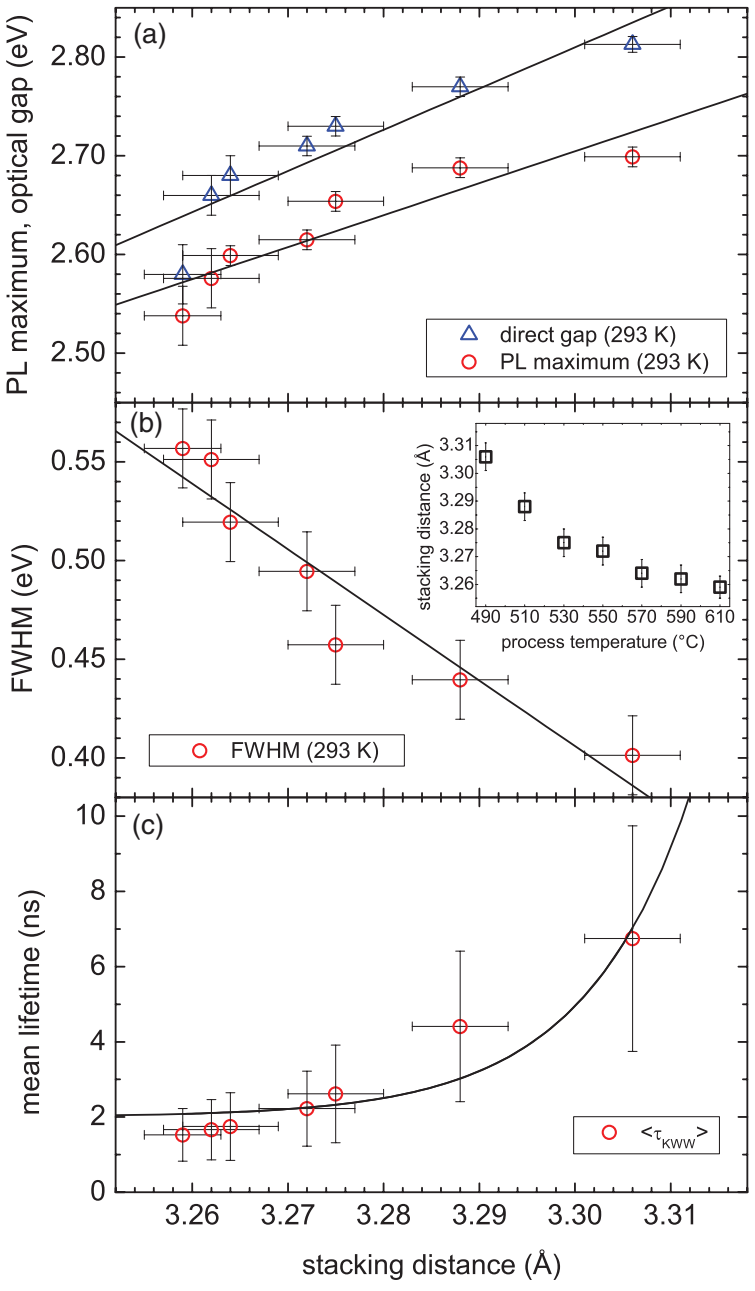

FIG. 9. (Color online) PL peak energy, direct optical transition energy (a), FWHM (b), and mean lifetime (c) as functions of the PCN stacking distance. The solid lines are fits according to a linear approach (a), (b), and an exponential function (c), respectively. The inset shows the dependence of stacking distance on the process temperature.

are bound via van der Waals forces along the $c$ direction. The main effect of higher process temperatures is qualitatively shown in Fig. 1(b). Depicted using Melem units, the increasing overlap of $\pi$ orbitals with decreasing stacking distance is evident. Note that the effect has been exaggerated for better visibility.

The energies of the direct (absorptive) opical transition, the PL emission maximum, and the FWHM are shown in Figs. 9(a) and 9(b), respectively. Based on the theory for molecular excitons on neighboring polymer strands, the transition energies for absorption and emission increase with $d_{\text {s }}$, until a saturation value is reached for infinite separation. ${ }^{33}$ On the other hand, increasing conjugation lengths lead to decreasing transition energies. ${ }^{33}$ For PCN, lacking aromatic connections between the monomer units, the conjugation length is essentially the intraplanar size of the tri- $s$-triazine unit $(\approx 7 \AA) .{ }^{11,34}$ At the same time, the excitonic coupling-which is proportional to the absorption/emission bandwidth-is expected to decrease with the distance, roughly as $d_{\mathrm{s}}^{-1} \cdot{ }^{27,28}$ Both these trends are tractable from our results. However, 
given the relative uncertainty of our experimental data, we have reverted to a linear approach, depicted by the solid lines in Figs. 9(a) and 9(b). The slopes for the optical gap, PL maximum, and FWHM are 4.2(8) eV/A, 3.2(6) eV/A, and $-3.3(5) \mathrm{eV} / \AA$, respectively. It is especially striking that the values extracted from our PL data yield essentially the same absolute slope. We may take this result as evidence for our assumption.

Figure 9(c) shows the distance-dependent mean lifetime of the PL signal. Following the hopping-transport hypothesis given by Lemmer and Göbel, the PL decay rate will decrease exponentially with the distance between hopping sites, in our case $d_{\mathrm{s}}{ }^{29}$ The resulting exponential increase of the mean lifetime may be observed in Fig. 9(c), although the resemblance is not as striking as in the process-temperature dependence (cf. Fig. 8). Consequently, the depicted exponential growth should rather be regarded as a guide to the eye than a fit yielding meaningful parameters.

Having discussed our experimental results regarding the presence of molecular singlet excitons, we may line out a qualitative model of the physical mechanism of optical excitation and relaxation in PCN.

(1) Upon irradiation with UV light, a molecular singlet exciton is generated, which is highly confined to the networkconstituting monomer (tri- $s$-triazine) due to nonaromatic connections within the polymer strand ${ }^{29}$ As a consequence of high exciton binding energies, a separation of the electron-hole pair is unlikely at room temperature, and particularly at cryogenic temperatures. ${ }^{11,29}$

(2) The transition energies of the absorption/emission process, as well as the excitonic coupling, depend on the cofacial distance (i.e., the distance perpendicular to the aromatic system) between mutual tri- $s$-triazine units on neighboring strands in a way characteristic for conjugated polymers. ${ }^{27,28,33}$

(3) Possible transport of the excitation via resonant energy transfer or hopping of excitons results in a strongly nonexponential decay of the PL signal. ${ }^{22,29,31}$ The transition rate for hopping transport is likely to be proportional to the (exponentially decreasing) overlap of the $\pi$ orbitals of cofacial aromatic systems. $^{29}$ In consequence, the lifetime of the PL decay grows exponentially with larger interplanar stacking distances in the polymer.

The model outlined here should in principle also be valid for PCN samples processed at lower temperature $\left(\theta \ll 500^{\circ} \mathrm{C}\right)$, since these materials consist also of the tri- $s$-triazine building block. ${ }^{4}$ However, since polycondensation does not occur for lower temperatures, these specimens resemble the molecular crystal Melem. ${ }^{9,10,12}$ Due to a different crystal structure with different cofacial distances between the aromatic units, the trends observed for the polycondensated samples are therefore not applicable, as seen in our results. Current investigations by the authors indicate that the crystal structure of PCN features a fairly complex behavior upon changing process temperature. Besides the prominent change in interplanar stacking distance $d_{\mathrm{s}},{ }^{4}$ mentioned in our discussion, further effects are found, including buckling of the polymer sheets, ${ }^{34}$ and distortions within the $a-b$ plane. ${ }^{10}$ These effects most likely also influence the optical properties, e.g., by affecting the cofacial distance and angle between mutual monomer units.

\section{CONCLUSIONS}

In conclusion, we have investigated the steady-state and transient photoluminescent properties of polymeric carbon nitride upon variation of sample temperature, excitation power, and process temperature. The obtained results differ substantially from those expected for a classical wide-band-gap semiconductor. Instead, we could successfully explain our results in the context of molecular singlet excitons, confined essentially to the tri-s-triazine unit of the polymer. This model approach was originally devised for conjugated polymers with conjugation lengths extending over several aromatic monomer units. Since PCN does not contain aromatic connections along its polymer strands, one may state that the material can be considered an optical quasimonomer.

Further insight to this debate is expected once PCN samples of optical quality are available, facilitating a quantitative determination of both linear and nonlinear optical measures.

\section{ACKNOWLEDGMENTS}

The authors would like to thank S. Levcenco for discussions concerning the PL of wide-band-gap semiconductors and I. Bochukov for calculations of the energy levels of Melem. Financial support by the German Bundesministerium für Bildung und Forschung (excellence cluster project "Light2Hydrogen," Grant No. 03IS2071F) is gratefully acknowledged. *christoph.merschjann@uni-rostock.de

${ }^{1}$ X. C. Wang, K. Maeda, A. Thomas, K. Takanabe, G. Xin, J. M. Carlsson, K. Domen, and M. Antonietti, Nat. Mater. 8, 76 (2009).

${ }^{2}$ F. Yang, M. Lublow, S. Orthmann, C. Merschjann, T. Tyborski, M. Rusu, S. Kubala, A. Thomas, R. Arrigo, M. Hävecker, and Th. Schedel-Niedrig, ChemSusChem 5, 1227 (2012).

${ }^{3}$ M. J. Bojdys, J.-O. Müller, M. Antonietti, and A. Thomas, Chem. Eur. J. 14, 8177 (2008)

${ }^{4}$ A. Thomas, A. Fischer, F. Goettmann, M. Antonietti, J.-O. Müller,

R. Schlögl, and J. M. Carlsson, J. Mater. Chem. 18, 4893 (2008).

${ }^{5}$ B. V. Lotsch, M. Döblinger, J. Sehnert, L. Seyfarth, J. Senker, O. Oeckler, and W. Schnick, Chem. Eur. J. 13, 4969 (2007).
${ }^{6}$ X. Li, J. Zhang, L. Shen, Y. Ma, W. Lei, Q. Cui, and G. Zou, Appl. Phys. A 94, 387 (2009).

${ }^{7}$ C. L. Schmidt and M. Jansen, J. Mater. Chem. 20, 4183 (2010).

${ }^{8}$ J. Liu, T. Zhang, Z. Wang, G. Dawson, and W. Chen, J. Mater. Chem. 21, 14398 (2011).

${ }^{9}$ B. Jürgens, E. Irran, J. Senker, P. Kroll, H. Müller, and W. Schnick, J. Am. Chem. Soc. 125, 10288 (2003).

${ }^{10}$ T. Tyborski, C. Merschjann, S. Orthmann, F. Yang, and Th. SchedelNiedrig (unpublished).

${ }^{11}$ M. N. Huda and J. A. Turner, J. Appl. Phys. 107, 123703 (2010).

${ }^{12}$ T. Tyborski, C. Merschjann, S. Orthmann, F. Yang, M.-Ch. Lux-Steiner, and Th. Schedel-Niedrig, J. Phys.: Condens. Matter 24, 162201 (2012). 
${ }^{13}$ Y. Zhang, T. Mori, J. Ye, and M. Antonietti, J. Am. Cem. Soc. 132, 6294 (2010).

${ }^{14}$ Y. Wang, X. C. Wang, and M. Antonietti, Ang. Chem. Int. Ed. 51, 68 (2012).

${ }^{15}$ F. Terenziani, C. Katan, E. Badaeva, S. Tretiak, and M. BlanchardDesce, Adv. Mater. 20, 4641 (2008).

${ }^{16}$ S. D. Phillips, R. Worland, G. Yu, T. Hagler, R. Freedman, Y. Cao, V. Yoon, J. Chiang, W. C. Walker, and A. J. Heeger, Phys. Rev. B 40, 9751 (1989).

${ }^{17}$ J. I. Pankove, Optical Processes in Semiconductors (Dover, New York, 1975).

${ }^{18}$ C. Merschjann, M. Mews, T. Mete, A. Karkatzinou, M. Rusu, B. V. Korzun, S. Schorr, P. Schubert-Bischoff, S. Seeger, Th. SchedelNiedrig, and M.-Ch. Lux-Steiner, J. Phys.: Condens. Matter 24, 175801 (2012).

${ }^{19}$ F. C. Spano, Acc. Chem. Res. 43, 429 (2009).

${ }^{20}$ G. Williams and D. C. Watts, Trans. Faraday Soc. 66, 80 (1970).

${ }^{21}$ F. Alvarez, A. Allegría, and J. Colmenero, Phys. Rev. B 44, 7306 (1991).

${ }^{22}$ C. Merschjann, M. Imlau, H. Brüning, B. Schoke, and S. Torbrügge, Phys. Rev. B 84, 052302 (2011).

${ }^{23}$ J. Clark, C. Silva, R. H. Friend, and F. C. Spano, Phys. Rev. Lett. 98, 206406 (2007).
${ }^{24}$ H. Bässler, Primary Photoexcitations in Conjugated Polymers: Molecular Exciton versus Semiconductor Band Model (World Scientific, Singapore, 1997), Chap. Excitons in Conjugated Polymers, pp. 51-98.

${ }^{25}$ W. Shan, X. C. Xie, J. J. Song, and B. Goldenberg, Appl. Phys. Lett. 67, 2512 (1995).

${ }^{26}$ F. C. Spano, J. Chem. Phys. 122, 234701 (2005).

${ }^{27}$ D. Beljonne, J. Cornil, R. Silbey, P. Millié, and J. L. Brédas, J. Chem. Phys. 112, 4749 (2000).

${ }^{28}$ W. J. D. Beenken and T. Pullerits, J. Chem. Phys. 120, 2490 (2004).

${ }^{29}$ U. Lemmer and E. O. Göbel, Primary Photoexcitations in Conjugated Polymers: Molecular Exciton versus Semiconductor Band Model (Ref. 24), Chap. Photoluminescence Spectroscopy as a Probe for Disorder and Excitonic Effects in Organic and Inorganic Semiconductors, pp. 211-253.

${ }^{30}$ H. Marciniak, X.-Q. Li, F. Würthner, and S. Lochbrunner, J. Phys. Chem. A 115, 648 (2011).

${ }^{31}$ T. Förster, Ann. Phys. (NY) 437, 55 (1948).

${ }^{32}$ F. Fennel and S. Lochbrunner, Phys. Rev. B 85, 094203 (2012).

${ }^{33}$ J. Cornil, D. A. dos Santos, X. Crispin, R. Silbey, and J. L. Brédas, J. Am. Chem. Soc. 120, 1289 (1998).

${ }^{34}$ J. Sehnert, K. Baerwinkel, and J. Senker, J. Phys. Chem. B 111, 10671 (2007). 